\title{
PAPER \\ Performance Evaluation of Beam Shapes in a Two-Step-Precoded Massive MIMO System**
}

\author{
Jumpei YAMAMOTO $^{\dagger *}$, Student Member, Toshihiko NISHIMURA ${ }^{\dagger}$, Senior Member, Takeo OHGANE ${ }^{\dagger a)}$, \\ Yasutaka OGAWA ${ }^{\dagger}$, Fellows, Daiki TAKEDA ${ }^{\dagger \dagger}$, Member, and Yoshihisa KISHIYAMA ${ }^{\dagger \dagger}$, Senior Member
}

\begin{abstract}
SUMMARY Massive MIMO is known as a promising technology for multiuser multiplexing in the fifth generation mobile communication system to accommodate the rapidly-increasing traffic. It has a large number of antenna elements and thus provides very sharp beams. As seen in hybrid beamforming, there have already been many papers on the concatenation of two precoders (beamformers). The inner precoder, i.e., a multi-beam former, performs a linear transformation between the element space and the beam space. The outer precoder forms nulls in the limited beam space spanned by selected beams to suppress the inter-user interference. In this two-step precoder, the beam shape is expected to determine the system performance. In this paper, we evaluate the achievable throughput performance for different beam-shaping schemes: a discrete Fourier transform (DFT) beam, Chebyshev weighted beams, and Taylor weighted beam. Simulations show that the DFT beam provides the best performance except the case of imperfect precoding and cell edge SNR of $30 \mathrm{~dB}$.

key words: 5G, massive MIMO, higher frequency band, two-step beamforming
\end{abstract}

\section{Introduction}

The use of super high frequency and extremely high frequency bands is planned for the fifth generation mobile communication system (5G). Since the free space path loss is proportional to the square of frequency, the path loss is severe and must be compensated. Thus, large or massive multiple-input multiple-output (MIMO) systems have been considered for 5G [2]-[5].

Hybrid beamforming, which is the concatenation of analog and digital precoders (beamformers), is a way to reduce the hardware and software complexity [6]-[9]. This concatenation can be generalized to a two-step precoder which is consist of a multi-beam former (first-step precoder) and a user-multiplexing precoder (second-step precoder). The first-step precoder transfers channels between transmit antennas and receive antennas from the element domain/space to the beam domain/space. In other words, the equivalent channel matrix, which is computed by multiplying the channel matrix by the multi-beam-forming matrix,

Manuscript received September 20, 2018.

Manuscript revised August 4, 2019.

Manuscript publicized December 9, 2019.

The authors are with the Graduate School of Information Science and Technology, Hokkaido University, Sapporo-shi, 0600814 Japan.

The authors are with NTT DOCOMO, INC., Yokosuka-shi, 239-8536 Japan.

* Presently, with NTT DOCOMO, INC., Japan.

**A part of this paper was presented at IEEE iWAT 2018 [1].

a)E-mail: ohgane@ist.hokudai.ac.jp

DOI: 10.1587/transcom.2018EBP3271

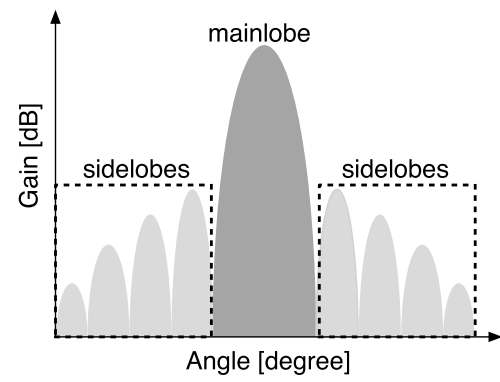

Fig. 1 A simple image of a beam shape of DFT-based beamforming.

expresses the channels between transmit beams and receive antennas. If some of the equivalent channels are quite low, we may not use those. For example, it has been reported that the number of clusters in millimeter bands is often very small as in [10]. Then, we can reduce the number of beams used in the second-step precoder for user-multiplexing to suppress implementation cost.

In the two-step precoder, there are several choices for the beamforming scheme in multi-beam forming. The most common one is phase-shifting (uniform excitation) as seen in discrete Fourier transform (DFT)-based beamforming. Figure 1 shows a simple image of a DFT beam shape. The beam produced by an antenna array generally has a mainlobe and sidelobes. It is known that the sidelobe levels are relatively high in the uniform excitation case. Non-uniform excitation such as Chebyshev distribution or Taylor distribution is a way to lower the sidelobe levels. However, nonuniform excitation degrades the gain and sharpness of the mainlobe in usual. To the best of the authors' knowledge, the impact of beam shape on the performance of the twostep precoder has not been discussed yet.

In this paper, we evaluate the performance dependency on the beam shape and the number of selected beams assuming a one-dimensional linear antenna array in a multiuser MIMO system where we use the block diagonalization (BD) [11], [12] for user multiplexing and the eigen mode transmission [13] in each user MIMO channel. For the beam shaping, we consider the Chebyshev weighted beam and the Taylor weighted beam in addition to the DFT beam.

The rest of the paper is organized as follows. In Sect. 2, we describe a two-step precoder. Three beam shapes and the beam selection rule in first-step precoding are explained in Sect. 3. In Sect. 4, the second-step precoding method and the corresponding achievable throughput formula are briefly in- 
troduced. Section 5 provides numerical evaluations including the precoding error due to time-variation of the channels and the paper is concluded in Sect. 6 .

\section{Two-Step Precoder}

In massive MIMO systems, the base station is equipped with a large number of antenna elements. Reducing the complexity of precoding (transmit beamforming) is one of the key implementation goals. Hybrid beamforming [6]-[9] is a well known technique to reduce the complexity of both software and hardware. It is concatenation of analog and digital precoders as shown in Fig. 2 where $N$ and $N_{\text {beam }}$ denote the numbers of transmit antennas and transmit beams, respectively. From the position of the precoders, the analog and digital precoders can be described as inner and outer precoders, respectively. Each antenna element is connected to the analog precoder (inner precoder) which has $N_{\text {beam }}$ input ports. And, these input ports are connected to the digital precoder (outer precoder) which achieves MIMO spatial multiplexing of $N_{\text {layer }}$ layers using the $N_{\text {beam }}$ beams.

The hybrid beamforming has $N_{\text {beam }}$ RF chains in the analog precoder. When $N_{\text {beam }}<N$, we can reduce the number of $\mathrm{D} / \mathrm{A}$ converters located at output ports of the digital precoder (and the number of RF amplifiers if they are located at the input ports of the analog precoder). The calculation complexity of digital precoding can also be reduced.

The analog precoder may have fixed multi-beams or variable beams via a phased array. If $N_{\text {beam-dimensional }}$ beam space is large enough and has a null space, we may select appropriate beams [6]-[9]. Then, the hybrid beamforming in Fig. 2 can be expressed as the general configuration shown in Fig. 3. Here, the analog precoder is replaced by a general precoder which may be either analog or digital. If a digital precoder is used at the first step, the hardware complexity cannot be reduced. However, we can still reduce the software complexity very much when the number of selected beams, $N_{s}$, is much smaller than $N_{\text {beam }}$. In this twostep precoder, the total beamforming matrix is expressed as $\mathbf{W}_{1} \mathbf{T} \mathbf{W}_{2}$ where $\mathbf{W}_{1}$ is an $N \times N_{\text {beam }}$ matrix for first-step precoding, $\mathbf{T}$ is an $N_{\text {beam }} \times N_{s}$ selection matrix, and $\mathbf{W}_{2}$ is an $N_{s} \times N_{\text {layer }}$ matrix for second-step precoding.

In general, uniform excitation is used in beamforming. Uniform excitation is simple and provides high directivity gain. Unfortunately, the sidelobe level becomes relatively large. The sidelobe may cause inter-layer interference if precoding is not perfect. Therefore, we test three beam shapes and discuss the dependency of the beam shape on the achievable throughput performance in Sect. 5 .

\section{First-Step (Inner) Precoding}

\subsection{Beam Generation}

The most general beamforming with uniform excitation is DFT-based one. In this paper, we consider an $N$-element uniform linear array with half-wavelength spacing ( $N$ is a

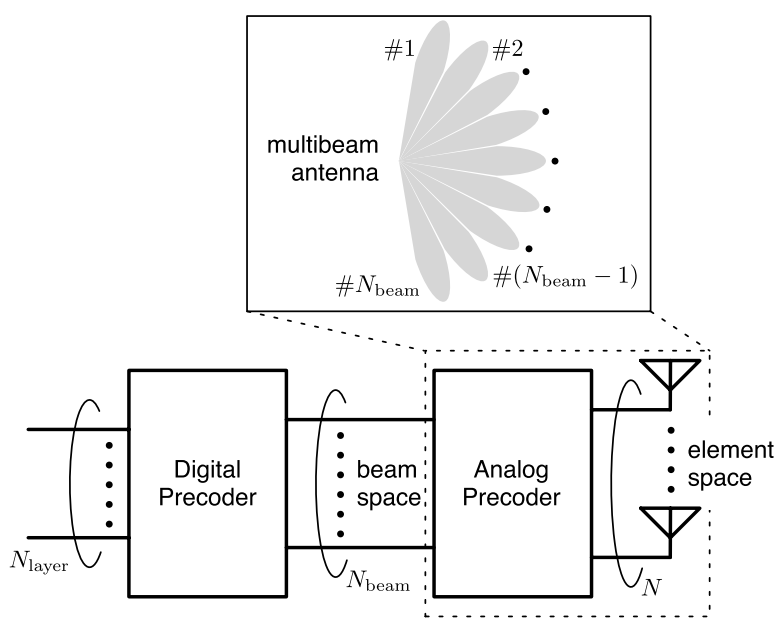

Fig. 2 Hybrid beamforming.

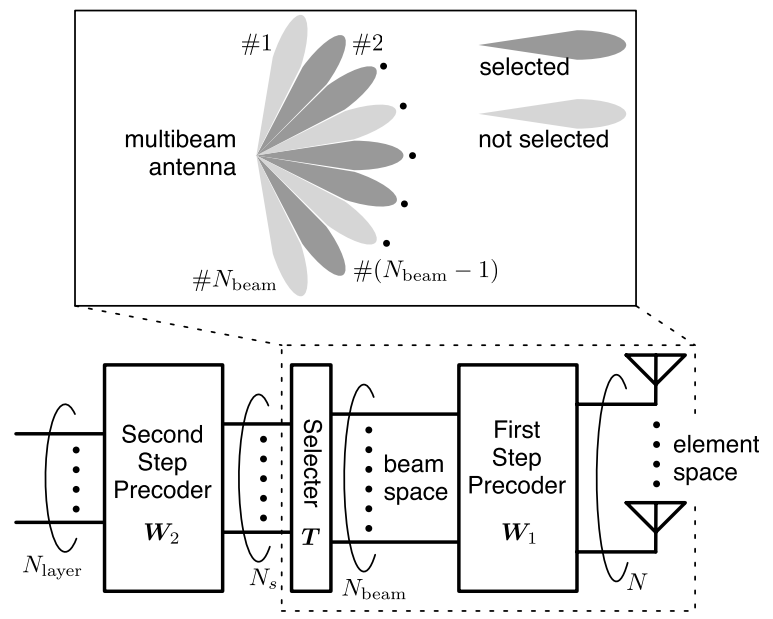

Fig.3 A generalized expression of hybrid forming.

power of 2). An $N \times N$ weight matrix of DFT-based beamforming is expressed as

$$
\mathbf{W}_{\mathrm{DFT}}=\frac{1}{\sqrt{N}}\left[\begin{array}{cccc}
w^{0} & w^{0} & \cdots & w^{0} \\
w^{0} & w^{1} & \cdots & w^{(N-1)} \\
w^{0} & w^{2} & \cdots & w^{2(N-1)} \\
\vdots & \vdots & \ddots & \vdots \\
w^{0} & w^{(N-1)} & \cdots & w^{(N-1)^{2}}
\end{array}\right],
$$

where $w$ represents $e^{-j 2 \pi / N}$. Using $\mathbf{W}_{\mathrm{DFT}}$ as $\mathbf{W}_{1}$, we can generate $N$ beams with $N$ elements, i.e., $N_{\text {beam }}=N$, and these beams are mutually orthogonal where each beam has nulls in the maximum gain direction of all other beams.

$2 N$-point DFT generates a $2 N \times 2 N$ DFT matrix where $w$ is replaced by $e^{-j 2 \pi / 2 N}$. Discarding the bottom $N$ rows of this matrix, we have an $N \times 2 N$ matrix $\mathbf{W}_{2 \mathrm{DFT}}$, i.e., $2 N$ different beams can be generated with an $N$-element array. The even column vectors of $\mathbf{W}_{2 \mathrm{DFT}}$ are phase-rotated one of each column vector of $\mathbf{W}_{\mathrm{DFT}}$ and equal to the column vectors of Butler matrix [14]. The direction of maximum gain of these vectors is shifted to the middle of DFT beams as shown in 


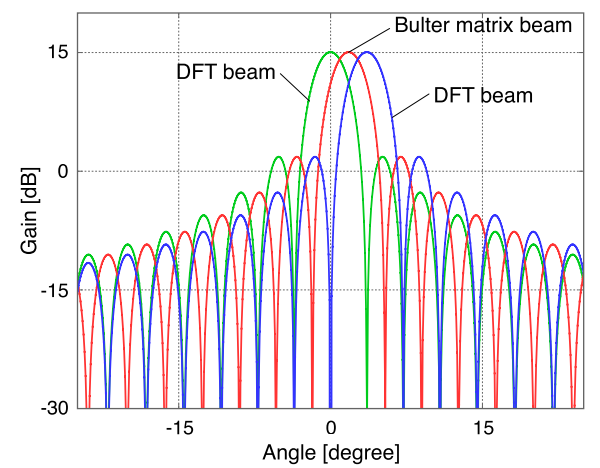

Fig. 4 Difference between DFT and Butler matrix beams. Note that only the first, second, and third beams of the oversampled DFT beam are shown.

Fig. 4. The sum of DFT and Butler matrix beams is called oversampled DFT beam [15].

Since each element of the DFT (and oversampled DFT) matrix has an equal amplitude, i.e., is uniformly excited, sidelobes become relatively large. In order to reduce the sidelobe levels, we consider two well-known excitation distributions.

The Chebyshev beam uses excitation according to Chebyshev distribution [16]. It can adjust the sidelobe levels to a certain value. The amplitude coefficients are symmetrical. The half of them, i.e., the $k$ th amplitude coefficient $(k=1,2, \ldots, N / 2)$ is expressed as

$$
I_{k}=A \sum_{q=k}^{M} \frac{(-1)^{M-q} z_{0}{ }^{2 q-1}(2 M-1)(q+M-2) !}{(q-k) !(q+k-1) !(M-q) !},
$$

where $M=N / 2$ and $A$ is a certain constant satisfying a condition that the sum of squares of the coefficients is one. $z_{0}$ is given by

$$
z_{0}=\frac{\left(r+\sqrt{r^{2}-1}\right)^{\frac{1}{2 M-1}}+\left(r-\sqrt{r^{2}-1}\right)^{\frac{1}{2 M-1}}}{2},
$$

where $r$ represents the ratio of mainlobe to sidelobe levels. Note that the sidelobes of Chebyshev beam have a constant level.

The other is Taylor beam using excitation according to Taylor distribution [17]. The $k$ th amplitude coefficient $(k=1,2, \ldots, N)$ is expressed as

$$
I_{k}=B\left(1+2 \sum_{m=1}^{n-1} F_{m} \cos \left[2 \pi m\left(\frac{k-1}{N-1}-\frac{1}{2}\right)\right]\right),
$$

where $B$ is a certain constant satisfying a condition that the sum of squares of the coefficients is one, and

$$
F_{m}=\frac{(-1)^{m+1}}{2 \prod_{\substack{p=1 \\ p \neq m}}^{n-1}\left(1-\frac{m^{2}}{p^{2}}\right)} \prod_{p=1}^{n-1}\left[1-\frac{m^{2}\left[C^{2}+\left(n-\frac{1}{2}\right)^{2}\right]}{n^{2}\left[C^{2}+\left(p-\frac{1}{2}\right)^{2}\right]}\right],
$$

where $C$ is a parameter related to the ratio of mainlobe to

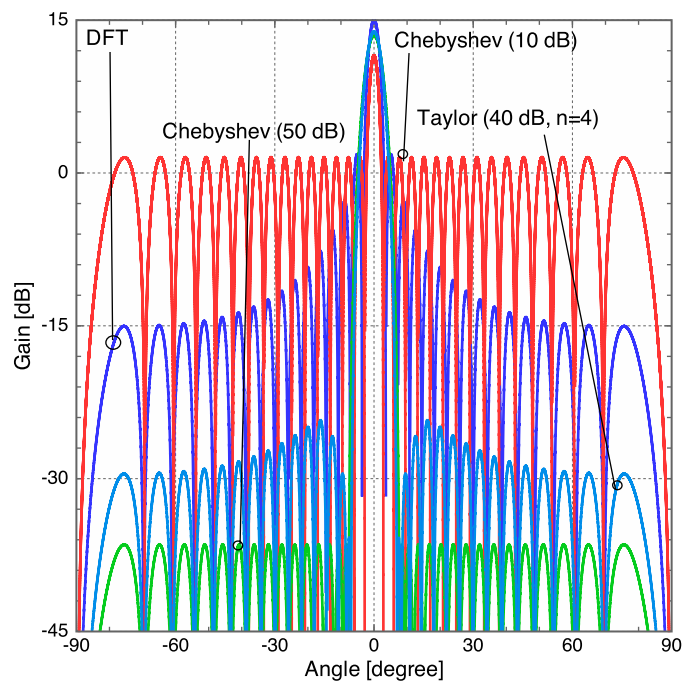

Fig. 5 Shapes of DFT, Chebyshev, and Taylor beams perpendicular to the array. Each $\mathrm{dB}$ value in the legend denotes the ratio of mainlobe to sidelobe levels.

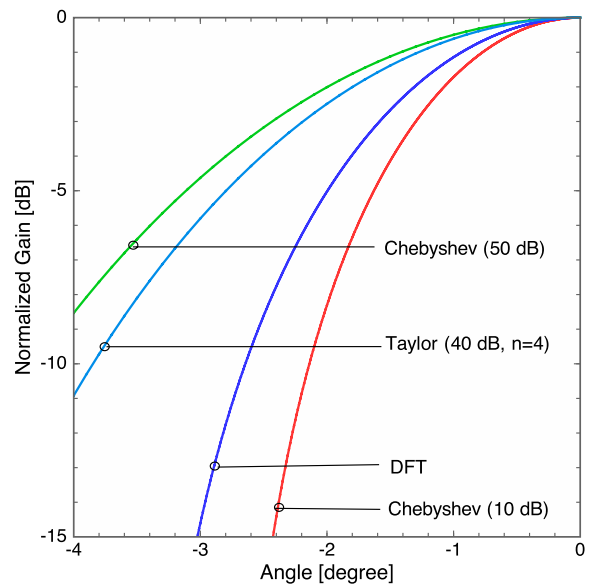

Fig. 6 Normalized mainlobes of DFT, Chebyshev, and Taylor beams perpendicular to the array.

sidelobe levels $r=20 \log (\cosh (\pi C))$. The sidelobe level of Tayler beam is not constant. $n$ controls the number of sidelobes which have almost the same level as $r$. When $n=$ 1 , the Tayler beam is equal to the DFT beam.

Figure 5 shows beam shapes of DFT beam, Chebyshev beam, and Taylor beam for $N=32$. In each weight matrix, the norm of each column vector is normalized to one. The loss of array gain at the zero degree of the Chebyshev and Taylor beams can be seen. Lowering the sidelobe level decreases the array gain and widens the mainlobe as shown in Fig. 6 where the maximum gain is normalized.

\subsection{Beam Space Transformation}

In the paper, we select $N_{s}$ beams form $N$ (or $2 N$ ) beams according to a certain rule which is described later. Once $N_{s}$ beams are selected, the precoding matrix is calculated for the given beams. In this paper, we assume that the num- 
ber of users is $N_{u}$ and that the number of receive antennas is equally set to $N_{r}$ for all users. Then, the $N_{u} N_{r} \times N$ channel matrix $\mathbf{H}$ is expressed as the stack of $k$ th user's channel matrix $\mathbf{H}_{k}$ of $N_{r} \times N$ dimensions for all users as $\mathbf{H}=\left[\mathbf{H}_{1}^{T} \mathbf{H}_{2}^{T} \cdots \mathbf{H}_{N_{u}}^{T}\right]^{T}$.

The effective channel matrix is obtained by multiplying $\mathbf{H}$ by an $N \times N_{s}$ selected beam matrix $\mathbf{W}_{s}$ which is constructed by $N_{s}$ column vectors selected out of the $N$ (or $2 N$ ) column vectors in the original beam matrix $\mathbf{W}_{1}$ (i.e., $\mathbf{W}_{\mathrm{DFT}}$, $\mathbf{W}_{\text {2DFT }}, \mathbf{W}_{\text {Chebyshev }}$, or $\left.\mathbf{W}_{\text {Tayler }}\right)$. When the indices of the selected column vectors in $\mathbf{W}_{1}$ are expressed as $l_{1}, \ldots, l_{N_{s}}$, the beam selection matrix is expressed by

$$
\mathbf{T}=\sqrt{\frac{N}{N_{s}}}\left[\mathbf{i}_{l_{1}} \cdots \mathbf{i}_{l_{N_{s}}}\right]
$$

where $\mathbf{i}_{j}$ is an $N$-dimensional (or $2 N$-dimensional) column vector with one at the $j$ th entry and zero elsewhere, and $\sqrt{N / N_{s}}$ is an adjustment coefficient for the constant transmission power. Finally, the first step precoding is achieved by

$$
\mathbf{W}_{s}=\mathbf{W}_{1} \mathbf{T}
$$

\subsection{Beam Selection Rule}

There are several ways to select the optimum or suboptimum beams from $N$ (or $2 N$ ) beams [6], [7], [9]. In this paper, we use a simple beam selection rule based on a concept measuring power which is expected to reach to each user [8]. First, the instantaneous power including path and shadowing losses, which are the squared norms of each column vector of $\mathbf{H}_{k} \mathbf{W}_{1}$, are ranked in descending order per user. Next, the beam of the highest rank is selected per user. When multiple users select the same beam at the highest rank, the user having higher received power selects the beam and the other users select the beam of the next rank. Then, we proceed the selection for the next rank and repeat it until the number of selected beams reaches to $N_{s}$.

\section{Second-Step (Outer) Precoding and Achievable Throughput}

In this paper, we use the block diagonalization [11], [12] to prevent the inter-user interference. The $\mathrm{BD}$ matrix $\mathbf{W}_{\mathrm{BD}}$ is calculated according to the effective channel matrix. Multiplying the $N_{s} \times N_{u} N_{r}$ BD matrix $\mathbf{W}_{\mathrm{BD}}$ by $\mathbf{H} \mathbf{W}_{s}$, we obtain the final equivalent channel

$$
\mathbf{H W}_{S} \mathbf{W}_{\mathrm{BD}}=\left[\begin{array}{ccc}
\tilde{\mathbf{H}}_{1,1} & \cdots & \tilde{\mathbf{H}}_{1, N_{u}} \\
\vdots & \ddots & \vdots \\
\tilde{\mathbf{H}}_{N_{u}, 1} & \cdots & \tilde{\mathbf{H}}_{N_{u}, N_{u}}
\end{array}\right] .
$$

The $k$ th digonal block $\tilde{\mathbf{H}}_{k, k}$ in (6) corresponds to the $k$ th user's single-user MIMO channel. The off-diagonal blocks are the zero matrix, i.e., $\tilde{\mathbf{H}}_{i, j}=\mathbf{O}$ for $i \neq j$ if no precoding errors.
In this paper, we evaluate the multiuser MIMO system from the viewpoint of achievable throughput assuming eigenmode multiplexing and a transmit power control based on the water-filling theory within each user. Here, we assume quasi-static Rayleigh fading during each frame. However, the above precoding may not be optimum to the channel when transmission delay is inevitable in time-varying environments. In such a case, inter-user interference occurs and thus $\tilde{\mathbf{H}}_{i, j} \neq \mathbf{O}$ for $i \neq j$. Then, the achievable user throughput [18] is expressed as

$$
\Gamma_{k}=\log _{2} \operatorname{det}\left(\mathbf{I}_{N_{r}}+\mathbf{R}_{k}^{-1} \tilde{\mathbf{H}}_{k, k} \mathbf{W}_{\mathrm{EM}, k} \mathbf{W}_{\mathrm{EM}, k}^{H} \tilde{\mathbf{H}}_{k, k}^{H}\right),
$$

where $\mathbf{W}_{\mathrm{EM}, k}$ is the $k$ th user's eigenmode precoding matrix [13] including the transmit power control and

$$
\mathbf{R}_{k}=\sum_{l=1, l \neq k}^{N_{u}} \tilde{\mathbf{H}}_{k, l} \mathbf{W}_{\mathrm{EM}, l} \mathbf{W}_{\mathrm{EM}, l}^{H} \tilde{\mathbf{H}}_{k, l}^{H}+\sigma^{2} \mathbf{I}_{N_{r}} .
$$

Here, $\sigma^{2}$ is the noise power at the user terminal. Again, $\tilde{\mathbf{H}}_{k, l}=\mathbf{O}$ for $k \neq l$ when no precoding errors. Then, $\mathbf{R}_{k}=$ $\sigma^{2} \mathbf{I}_{N_{r}}$. Finally, the second-step precoding matrix is given by

$$
\mathbf{W}_{2}=\mathbf{W}_{\mathrm{BD}} \operatorname{diag}\left(\mathbf{W}_{\mathrm{EM}, 1} \mathbf{W}_{\mathrm{EM}, 2} \cdots \mathbf{W}_{\mathrm{EM}, N_{u}}\right) \text {. }
$$

\section{Numerical Evaluation}

\subsection{Simulation Conditions}

Figure 7 shows the user distribution and the scatterer distribution for channel modeling. Table 1 describes the simulation parameters used in this paper. The base station has a linear antenna array on the $x$ axis where the element spacing is set to half wavelength. The user is uniformly distributed within a $120^{\circ}$ sector cell, and 13 scattering-points are located on a circle around each user. The angular spread of the scattering circle at the base station is denoted by $\Delta \theta$, and it is assumed that $\Delta \theta$ is equal for all the users.

Rayleigh fading is simulated by the sum of signals arriving via these scattering points (Jakes model). Also, it is

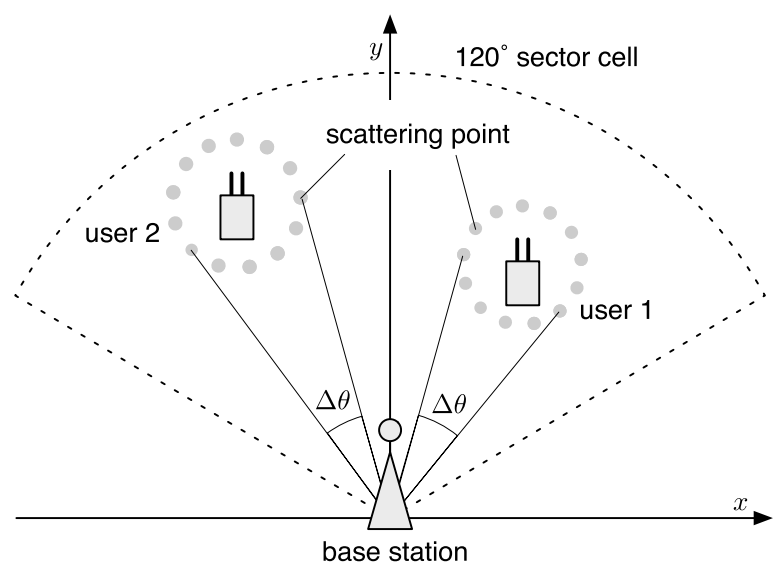

Fig. 7 User and scatterer distributions. 
Table 1 Simulation parameters.

\begin{tabular}{c|c}
\hline Number of transmit antennas & $N=32$ \\
\hline Total number of transmit beams & $N_{\text {beam }}=32,64$ \\
\hline Number of selected beams & $N_{s}=8,12, \ldots, N_{\text {beam }}$ \\
\hline Number of users & $N_{u}=4$ \\
\hline Number of receive antennas & $N_{r}=2$ \\
\hline Number of scattering points & 13 \\
\hline Angular spread & $\Delta \theta=5^{\circ}, 20^{\circ}$ \\
\hline Cell edge SNR & $-5,10,30 \mathrm{~dB}$ \\
\hline Sector size & $-60^{\circ} \sim 60^{\circ}$ \\
\hline User location distribution & Uniform within the sector \\
\hline Channel statistics & Quasi-static Rayleigh fading \\
\hline Normalized Doppler frequency & $f_{D} \tau=0,0.01$ \\
\hline Path loss exponent & 3.0 \\
\hline Shadowing standard deviation & $7.0 \mathrm{~dB}$ \\
\hline
\end{tabular}

assumed that the distance between the receiving antennas of each user is sufficiently long, and thus the fading is assumed to be uncorrelated. For transmission precoding and beam selection, it is assumed that channel information at $t=0$ can be perfectly known at the base station. However, when the transmission processing delay $\tau$ is not negligible, the transmit-weight matrix no longer match the channels at $t=\tau$. The average user throughput is calculated in 100 different fading environments changing phases of the scatterers at each user position in the above environment with or without the processing delay. In addition, the location is changed 1,000 times to obtain the cumulative distribution of the achievable throughput.

\subsection{Beam Shape Dependency}

Figure 8 shows the 10,50 , and $90 \%$ values of the cumulative probability distribution of user throughput for each beam shape without the oversampled DFT $\left(N_{\text {beam }}=32\right)$ when normalized Doppler frequency, $f_{D} \tau$, is 0 , i.e., the case without precoding errors. The angular spread is set to $5^{\circ}$ in Fig. 8(a) and $20^{\circ}$ in Fig. 8(b).

For all cumulative probability, the DFT beam showed the highest throughput. Chebyshev beams and Taylor beam have lower sidelobes. In the no inter-user interference scenarios, however, the user throughput is not affected by the sidelobe level. In contrast, the wider mainlobe increases the channel correlation between adjacent beams due to larger inter-beam overlapping. In fact, low singular values can be seen in the case of Chebyshev and Taylor beams whereas the distribution of singular values is flat for the DFT beam as shown in Fig. 9. On the other hand, the loss of array gain in Chebyshev and Taylor beams directly decreases the user throughput. Consequently, the DFT beam was the best. This tendency seems to be not sensitive to the angular spread.

\subsection{Dependency of Number of Selected Beams}

The case of $N_{s}=8=N_{u} N_{r}$ gave the highest throughput

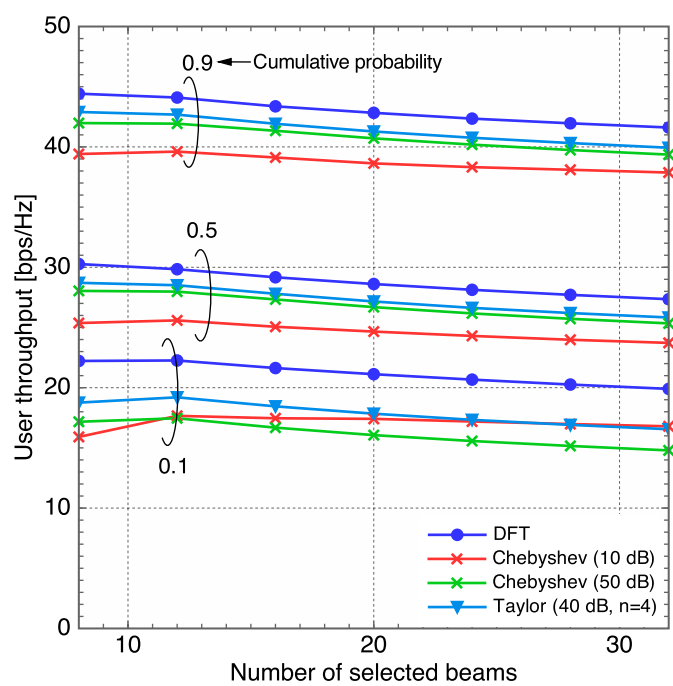

(a) $\Delta \theta=5^{\circ}$

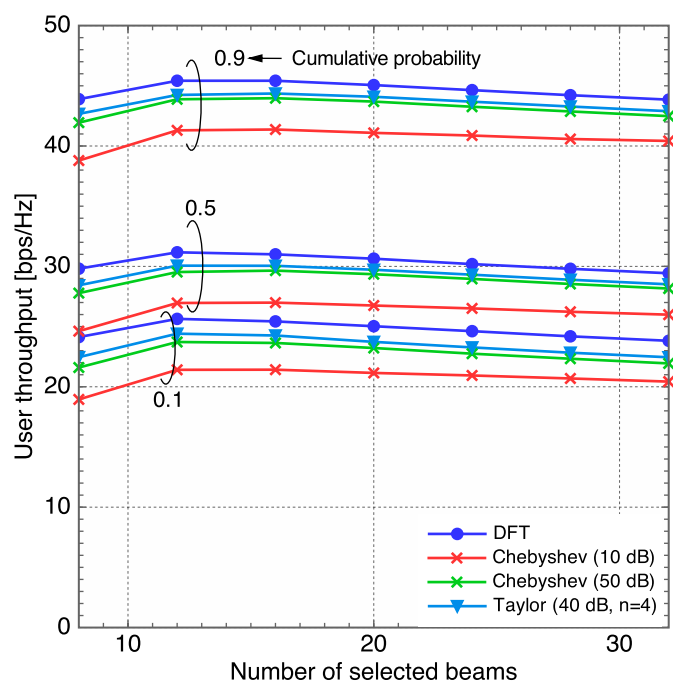

(b) $\Delta \theta=20^{\circ}$

Fig. 810,50 , and $90 \%$ values of user throughput where $N=32, N_{\text {beam }}=$ $32, f_{D} \tau=0$, and the cell edge $\mathrm{SNR}=30 \mathrm{~dB}$. Each $\mathrm{dB}$ value in the legend denotes the ratio of mainlobe to sidelobe levels.

in the DFT beam for $\Delta \theta=5^{\circ}$ as shown in Fig. 8(a). If we select too many beams, some of those include only the weak signals. The weak signals are consistently regarded as the interference in the BD whereas these barely contribute to the user throughput. Many nulls are needed to suppress the interference perfectly which significantly degrades the BD gain. When $\Delta \theta=20^{\circ}$, however, we need a larger number of selected beam due to the spatial correlation of channels between the adjacent beams as shown in Fig. 8(b).

\subsection{Oversampled DFT}

Figure 10 indicates the case with the oversampled DFT, i.e., $N_{\text {beam }}=2 N=64$. In comparison to Fig. 8 , the oversampled DFT beam slightly increases the user throughput because 


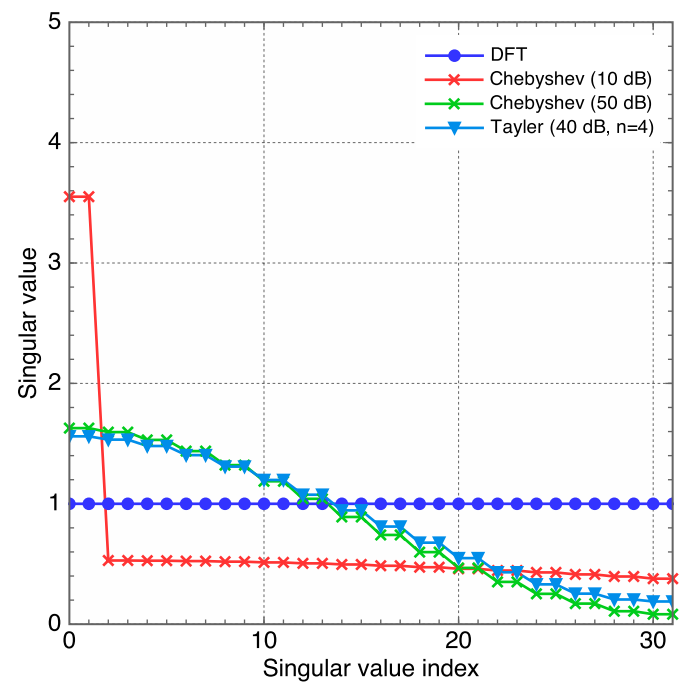

Fig. 9 Singular value distributions for $32 \times 32$ weight matrices of DFT, Chebyshev, and Tayler beams.

the sector cell is covered densely as shown in Fig. 4. On the other hand, it needs a larger number of selected beams to obtain the highest throughput. This is due to the spatial correlation property similar to the case for $N_{\text {beam }}=32$ and $\Delta \theta=20^{\circ}$. Although it is necessary to pay attention to the complexity, we focus into the oversampled DFT beam to obtain the higher throughput in the followings.

\subsection{Precoding Errors in Time-Varying Environments}

Next, let us consider the processing delay $\tau$. Then, the interuser interference occurs due to that the precoder matches the past channel and thus causes errors in the BD for the current channel. Such evaluation may also be useful when we expect the impacts of channel estimation errors, inaccurate channel feedback, and/or calibration errors of RF components. Figures 11, 12, and 13 show the cases for $f_{D} \tau=0.01$. The cell edge SNR values are set to 30,10 , and $-5 \mathrm{~dB}$, respectively.

The comparison of Fig. 11 with Fig. 10, where only $f_{D} \tau$ is different, clearly indicates that the user throughput performance degrades due to the outdated precoding and that the degradation is larger in the case for $\Delta \theta=20^{\circ}$ since the temporal correlation between the past and current channels for each single beam becomes lower. The degradation is severer for the $90 \%$ value of the DFT beam and Chebyshev beam of $r=10 \mathrm{~dB}$. These beams have higher sidelobes as shown in Fig. 5. Thus, the inter-user interference caused by imperfect precoding becomes much larger. For the 50\% and 10\% values, however, the degradation is not so severe. It is supposed that the inter-user interference can be decreased by these beams to almost the noise level for such middle or low quality users.

Although the highest throughput is still obtained by the DFT beam for $10 \%$ and $50 \%$ values, the Chebyshev beam of $r=50 \mathrm{~dB}$ provides the highest throughput for $90 \%$ value.

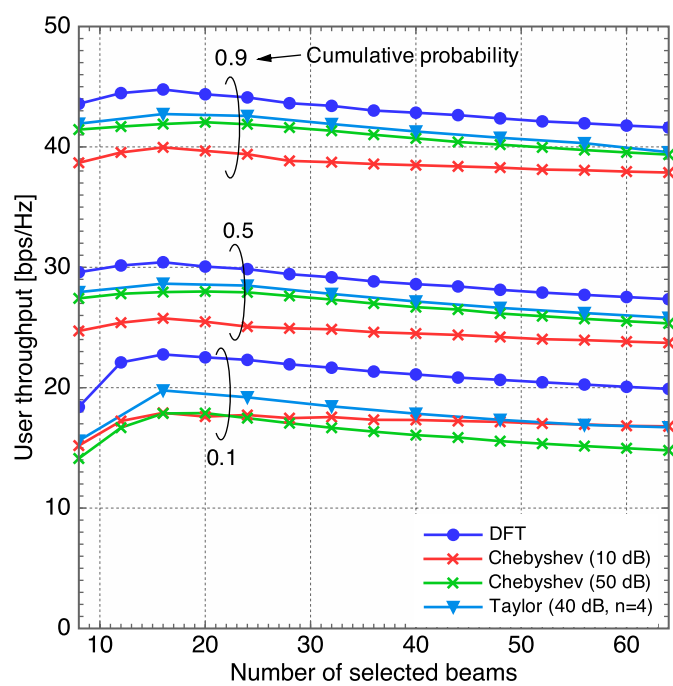

(a) $\Delta \theta=5^{\circ}$

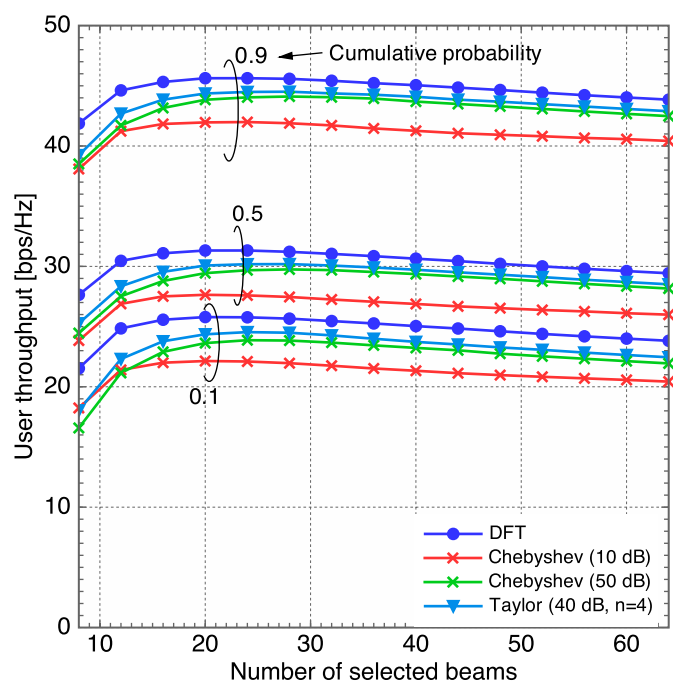

(b) $\Delta \theta=20^{\circ}$

Fig. 1010,50 , and $90 \%$ values of user throughput where $N=32$ and $N_{\text {beam }}=64$, i.e., the oversampled case. $f_{D} \tau=0$ and the cell edge SNR is set to $30 \mathrm{~dB}$. Each $\mathrm{dB}$ value in the legend denotes the ratio of mainlobe to sidelobe levels.

Specifically, the degradation for the maximum throughput is about $10 \mathrm{bps} / \mathrm{Hz}$ for the DFT beam and less than $4 \mathrm{bps} / \mathrm{Hz}$ for the Chebyshev beam of $r=50 \mathrm{~dB}$. It is supposed that the inter-user interference due to the precoding errors can be reduced by the lower sidelobes. Thus, if we need to minimize the peak throughput degradation, a beam with low sidelobes maybe the best choice.

When the cell edge SNR is decreased as in Figs. 12 and 13 , the DFT beam provides the best performance again even for $90 \%$ value. In these cases, the noise level is much higher than the case of Fig. 11. Therefore, the inter-user interference caused by imperfect precoding is not so impactive. In addition, it is important for performance improvement in such severe SNR conditions to increase the signal power and 


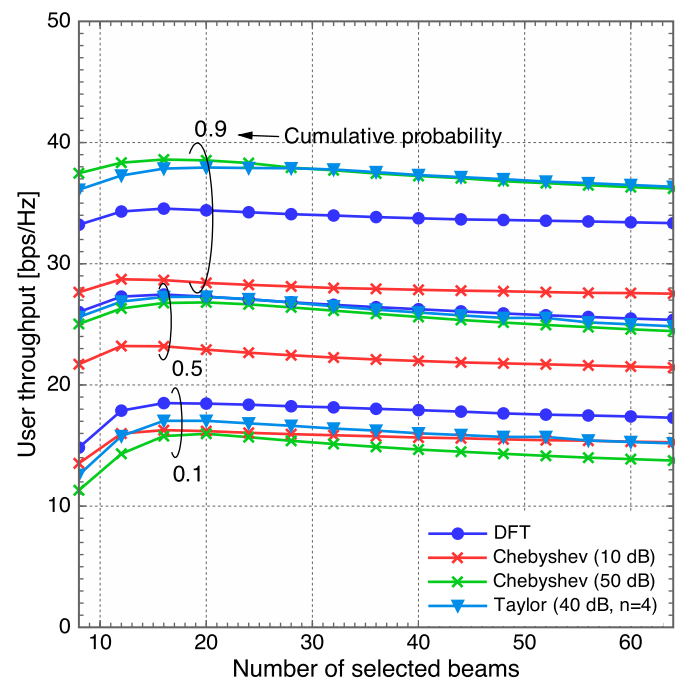

(a) $\Delta \theta=5^{\circ}$

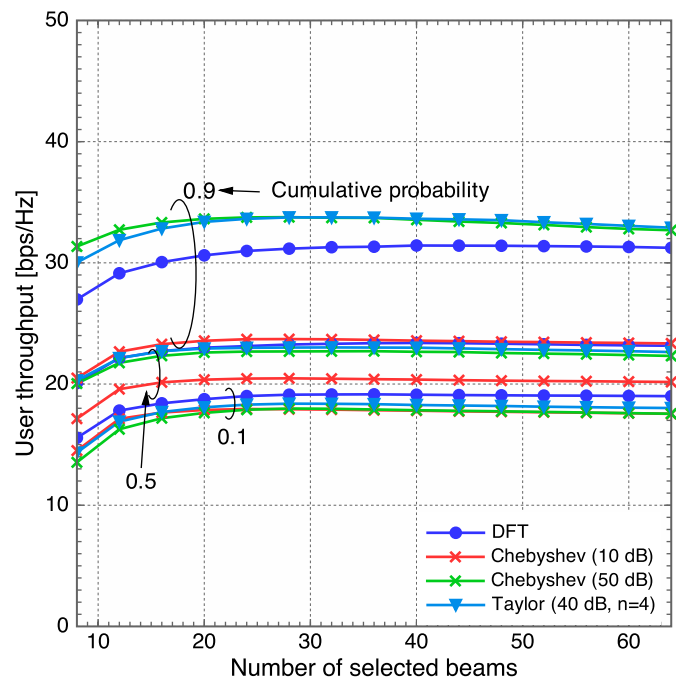

(b) $\Delta \theta=20^{\circ}$

Fig. 11 10, 50, and 90\% values of user throughput where $f_{D} \tau=0.01$, i.e., the imperfect precoding case. $N=32, N_{\text {beam }}=64$, and the cell edge SNR is set to $30 \mathrm{~dB}$. Each $\mathrm{dB}$ value in the legend denotes the ratio of mainlobe to sidelobe levels.

rank of the effective channel matrix using low inter-beamcorrelated beams as discussed in 5.2. Consequently, it can be said that the DFT beam is really robust.

\section{Conclusions}

In this paper, we we evaluated the impact of beam shape on two-step precoding in large scale time-invariant/variant multiuser MIMO environments. As imperfect precoding creates inter-user interference, low sidelobes are preferable basically. Unfortunately, lowering the sidelobe level causes a loss of the maximum gain and widens the mainlobe. Simulations showed that DFT beam forming yielded the best performance in most cases. The Chebyshev beam with $r=50 \mathrm{~dB}$, the sidelobe level of which is $50 \mathrm{~dB}$ less than

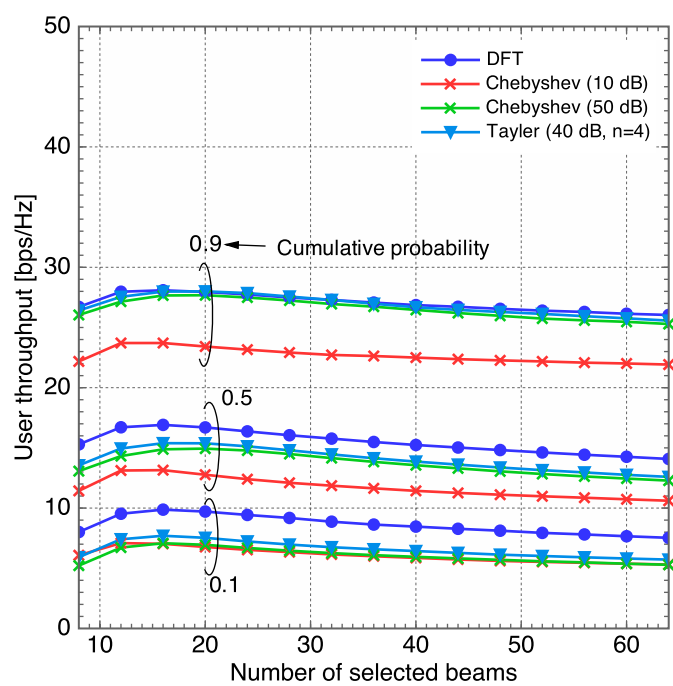

(a) $\Delta \theta=5^{\circ}$

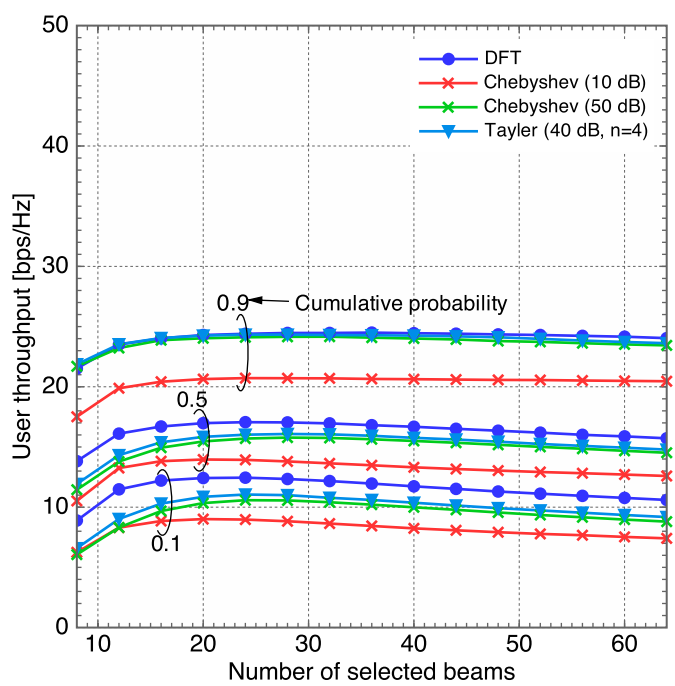

(b) $\Delta \theta=20^{\circ}$

Fig. 1210,50 , and $90 \%$ values of user throughput where $f_{D} \tau=0.01$, i.e., the imperfect precoding case. $N=32, N_{\text {beam }}=64$, and the cell edge SNR is set to $10 \mathrm{~dB}$. Each $\mathrm{dB}$ value in the legend denotes the ratio of mainlobe to sidelobe levels.

the mainlobe level, has been the best for the good users' throughputs when the precoding error is not ignorable and the SNR condition is very high. Considering that such a scenario is very limited, we can conclude that the DFT beam is robust as the inner beamformer.

It should also be noted that the number of selected beams has the optimum value when we use the BD for user multiplexing. The beams, the transmit power of which does not reach to (or barely reaches to) the receivers, do not contribute to the throughput gain but still require to form nulls each other in the BD. Thus, the gain at the direction of a certain target user may be decreased when null positions are closely located. 


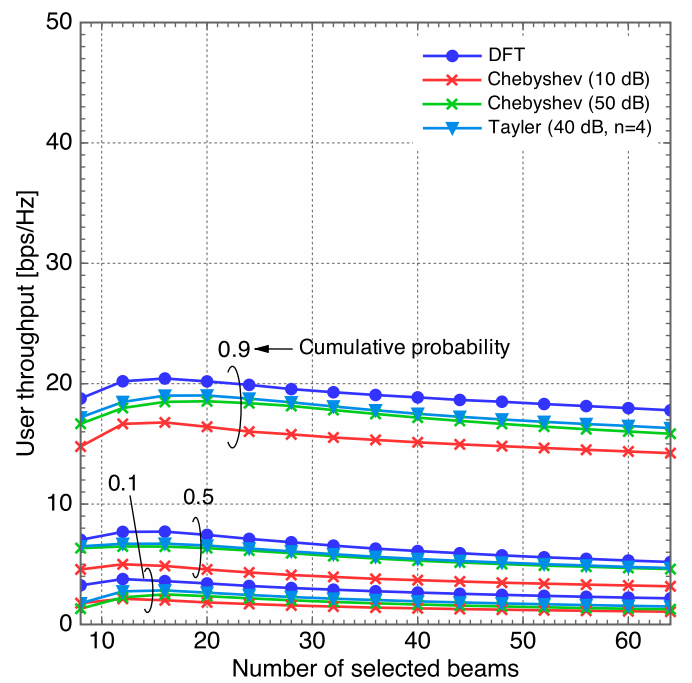

(a) $\Delta \theta=5^{\circ}$

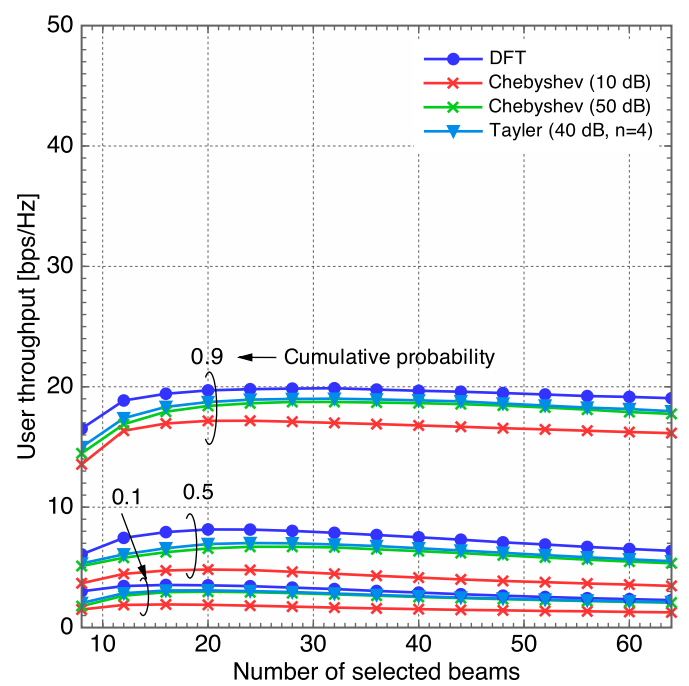

(b) $\Delta \theta=20^{\circ}$

Fig. 1310,50 , and $90 \%$ values of user throughput where $f_{D} \tau=0.01$, i.e., the imperfect precoding case. $N=32, N_{\text {beam }}=64$, and the cell edge SNR is set to $-5 \mathrm{~dB}$. Each $\mathrm{dB}$ value in the legend denotes the ratio of mainlobe to sidelobe levels.

\section{References}

[1] J. Yamamoto, T. Nishimura, T. Ohgane, Y. Ogawa, D. Takeda, and Y. Kishiyama, "Performance evaluation on beam shapes of a large MIMO system," Proc. IEEE iWAT 2018, March 2018.

[2] E.G. Larsson, O. Edfors, F. Tufvesson, and T.L. Marzetta, "Massive MIMO for next generation wireless systems," IEEE Commun. Mag., vol.52, no.2, pp.186-195, Feb. 2014.

[3] F. Rusek, D. Persson, B.K. Lau, E.G. Larsson, T.L. Maretta, O. Edfors, and F. Tufvesson, "Scaling up MIMO: Opportunities and challenges with very large arrays," IEEE Signal Process. Mag., vol.30, no.1, pp.40-60, Jan. 2013.

[4] T.L. Marzetta, "Noncooperative cellular wireless with unlimited numbers of base station antennas," IEEE Trans. Wireless Commun., vol.9, no.11, pp.3590-3600, Nov. 2010.

[5] T.S. Rappaport, S. Sun, R. Mayzus, H. Zhao, Y. Azar, K. Wang,
G.N. Wong, J.K. Schulz, M. Samimi, and F. Gutierrez, "Millimeter wave mobile communications for 5G cellular: It will work!," IEEE Access, vol.1, pp.335-349, May 2013.

[6] T. Kim, J. Park, J.Y. Seol, S. Jeong, J. Cho, and W. Roh, "Tens of Gbps support with mmWave beamforming systems for next generation communications," Proc. IEEE GLOBECOM 2013, pp.36853690, Dec. 2013.

[7] A. Alkhateeb, J. Mo, N.G. Prelcic, and R.W. Heath, "MIMO precoding and combining solutions for millimeter-wave systems," IEEE Commun. Mag., vol.52, no.12, pp.122-131, Dec. 2014.

[8] T. Obara, S. Suyama, J. Shen, and Y. Okumura, "Joint processing of analog fixed beamforming and CSI-based precoding for super high bit rate massive MIMO transmission using higher frequency bands," IEICE Trans. Commun., vol.E98-B, no.8, pp.1474-1481, Aug. 2015

[9] A.F. Molisch, V.V. Ratnam, S. Han, Z. Li, S.L. H. Nguyen, L. Li, and K. Haneda, "Hybrid beamforming for massive MIMO: A survey," IEEE Commun. Mag., vol.55, no.9, pp.134-141, Sep. 2017.

[10] X. Zhao, S. Li, Q. Wang, M. Wang, S. Sun, and W. Hong, "Channel measurements, modeling, simulation and validation at $32 \mathrm{GHz}$ in outdoor microcells for 5G radio systems," IEEE Access, vol.5, pp.1062-1072, 2017.

[11] Q.H. Spencer, A.L. Swindlehurst, and M. Haardt, "Zero-forcing methods for downlink spacial multiplexing in multiuser MIMO channels," IEEE Trans. Signal Process., vol.52, no.2, pp.461-471, Jan. 2004.

[12] K. Nishimori, T. Hiraguri, and H. Makino, "Transmission rate by user antenna selection for block diagonalization based multiuser MIMO system," IEICE Trans. Commun., vol.E97-B, no.10, pp.2128-2126, Oct. 2014.

[13] K. Miyashita, T. Nishimura, T. Ohgane, Y. Ogawa, Y. Takatori, and C. Keizo, "High data-rate transmission with eigenbeam-space division multiplexing (E-SDM) in a MIMO channel," Proc. IEEE VTC 2002-Fall, vol.3, pp.1302-1306, Sept. 2002.

[14] T.N. Kaifas and J.N. Sahalos, "On the design of a single-layer wideband butler matrix for switched-beam UMTS system applications," IEEE Antennas Propag. Mag., vol.48, no.6, pp.193-204, Dec. 2006.

[15] Y. Xie, S. Jin, Y. Zhu, X. Gao, and Y. Huang, "A limited feedback scheme for 3D multiuser MIMO based on Kronecker product codebook," Proc. IEEE PIMRC 2013, pp.1130-1135, Sept. 2013.

[16] D. Barbiere, "A method for calculating the current distribution of Tschebyscheff arrays," Proc. IRE., vol.40, no.1, pp.78-82, Jan. 1952

[17] F. Tseng, "Design of array and line-source antennas for Taylor patterns with a null," IEEE Trans. Antennas Propag., vol.AP-27, no.4, pp.474-479, July 1979.

[18] Y. Song and S.D. Blostein, "MIMO channel capacity in co-channel interference," Proc. 21st Biennial Symposium on Communications, pp.220-224, June 2002.

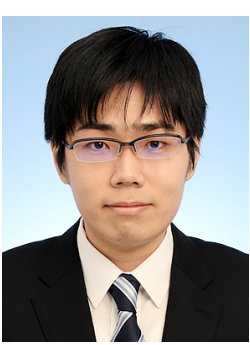

Jumpei Yamamoto received the B.E. degree in electronics and information engineering from Kagawa University, Takamatsu, Japan, in 2016 and the M.I.S. degree in information science from Hokkaido University, Sapporo, Japan, in 2018. Since 2018, he has been with NTT DOCOMO, Inc. His interests are in MIMO systems and mobile communications 


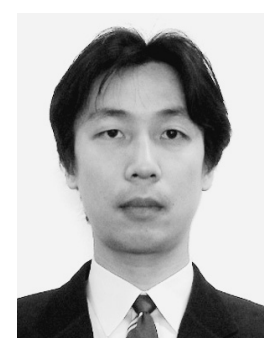

Toshihiko Nishimura received the B.S. and M.S. degrees in physics, and the Ph.D. degree in electronics engineering from Hokkaido University, Sapporo, Japan, in 1992, 1994, and 1997, respectively. Since 1998, he has been with Hokkaido University, where he is currently an Associate Professor. His current research interests are in MIMO systems using smart antenna techniques. He received the Young Researchers' Award of IEICE Japan in 2000, the Best Paper Award from IEICE Japan in 2007, TELECOM system technology award from the Telecommunications Advancement Foundation of Japan in 2008, the Best Magazine Paper Award from the IEICE Communications Society in 2011, and the Best Tutorial Paper (in Japanese) Award from the IEICE Communications Society in 2018. He is a member of the IEEE.

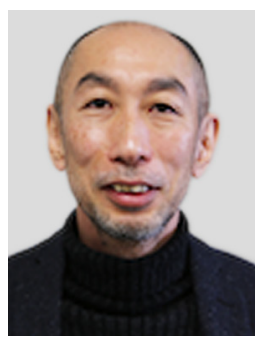

Takeo Ohgane received the B.E., M.E., and $\mathrm{Ph} . \mathrm{D}$. degrees in electronics engineering from Hokkaido University, Sapporo, Japan, in 1984, 1986, and 1994, respectively. He was with the Communications Research Laboratory of the Ministry of Posts and Telecommunications from 1986 to 1992 . He was on assignment at the ATR Optical and Radio Communications Research Laboratory from 1992 to 1995. Since 1995, he has been with Hokkaido University, where he is an Associate Professor. He was at the Centre for Communications Research of the University of Bristol, U.K., as a Visiting Fellow in 2005-2006. His interests are in MIMO signal processing for wireless communications. He received the Young Researchers' Award of IEICE Japan in 1990, the IEEE AP-S Tokyo Chapter Young Engineer Award in 1993, the Best Paper Award from IEICE Japan in 2007, TELECOM system technology award from the Telecommunications Advancement Foundation of Japan in 2008, the Best Magazine Paper Award from the IEICE Communications Society in 2011, and the Best Tutorial Paper (in Japanese) Award from the IEICE Communications Society in 2018. He is a member of the IEEE.

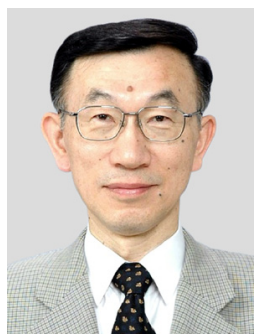

Yasutaka Ogawa received the B.E., M.E., and Ph.D. degrees from Hokkaido University, Sapporo, Japan, in 1973, 1975, and 1978, respectively. Since 1979, he has been with Hokkaido University, where he is currently a Professor Emeritus at the Graduate School of Information Science and Technology. During 19921993, he was with the ElectroScience Laboratory of Ohio State University, U.S.A., as a Visiting Scholar, on leave from Hokkaido University. His interests are in adaptive antennas, mobile communications, super-resolution techniques, and MIMO systems. He received the Yasujiro Niwa outstanding paper award in 1978, the Young Researchers' Award of IEICE Japan in 1982, the Best Paper Award from IEICE Japan in 2007, TELECOM system technology award from the Telecommunications Advancement Foundation of Japan in 2008, the Best Magazine Paper Award from the IEICE Communications Society in 2011, the Achievement Award from IEICE in 2014, and the Best Tutorial Paper (in Japanese) Award from the IEICE Communications Society in 2018. He is a Fellow of the IEEE.

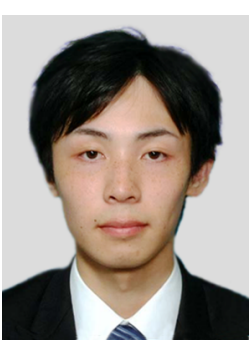

Daiki Takeda received the B.S. and M.S. degrees from Hokkaido University, Sapporo, Japan, in 2010 and 2012, respectively. In 2012, he joined NTT DOCOMO, INC. Since joining NTT DOCOMO he is engaged in research and development on $4 \mathrm{G} / 5 \mathrm{G}$ radio access technologies and physical layer standardization in 3GPP

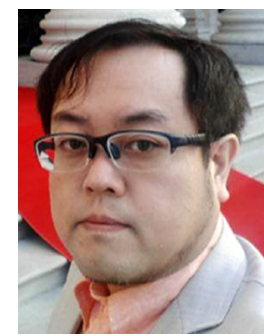

Yoshihisa Kishiyama is a Manager of 5G Laboratories in NTT DOCOMO, INC. He received his B.E., M.E., and Ph.D. degrees from Hokkaido University, Sapporo, Japan in 1998, 2000 , and 2010, respectively. Since he joined NTT DOCOMO in 2000, he has been involved in research and development on $4 \mathrm{G} / 5 \mathrm{G}$ radio access technologies and physical layer standardization in 3GPP. In 2012, he received the International Telecommunication Union Association of Japan (ITU-AJ) Award for contributions to

LTE. 